\title{
DDFlow: Learning Optical Flow with Unlabeled Data Distillation
}

\author{
Pengpeng Liu, ${ }^{\dagger *}$ Irwin King, ${ }^{\dagger}$ Michael R. Lyu, ${ }^{\dagger} \mathbf{J i a ~ X u}^{\S}$ \\ ${ }^{\dagger}$ The Chinese University of Hong Kong, Shatin, N.T., Hong Kong \\ $\S$ Tencent AI Lab, Shenzhen, China \\ \{ppliu, king, lyu\}@cse.cuhk.edu.hk, jiajxu@tencent.com
}

\begin{abstract}
We present DDFlow, a data distillation approach to learning optical flow estimation from unlabeled data. The approach distills reliable predictions from a teacher network, and uses these predictions as annotations to guide a student network to learn optical flow. Unlike existing work relying on handcrafted energy terms to handle occlusion, our approach is data-driven, and learns optical flow for occluded pixels. This enables us to train our model with a much simpler loss function, and achieve a much higher accuracy. We conduct a rigorous evaluation on the challenging Flying Chairs, MPI Sintel, KITTI 2012 and 2015 benchmarks, and show that our approach significantly outperforms all existing unsupervised learning methods, while running at real time.
\end{abstract}

\section{Introduction}

Optical flow estimation is a core computer vision building block, with a wide range of applications, including autonomous driving (Menze and Geiger 2015), object tracking (Chauhan and Krishan 2013), action recognition (Simonyan and Zisserman 2014) and video processing (Bonneel et al. 2015). Traditional approaches (Horn and Schunck 1981; Brox et al. 2004; Brox and Malik 2011) formulate optical flow estimation as an energy minimization problem, but they are often computationally expensive (Xu, Ranftl, and Koltun 2017). Recent learning-based methods (Dosovitskiy et al. 2015; Ranjan and Black 2017; Ilg et al. 2017; Hui, Tang, and Loy 2018; Sun et al. 2018) overcome this issue by directly estimating optical flow from raw images using convolutional neural networks (CNNs). However, in order to train such CNNs with high performance, it requires a large collection of densely labeled data, which is extremely difficult to obtain for real-world sequences.

One alternative is to use synthetic datasets. Unfortunately, there usually exists a large domain gap between the distribution of synthetic images and natural scenes (Liu et al. 2008). Previous networks (Dosovitskiy et al. 2015; Ranjan and Black 2017) trained only on synthetic data turn to overfit, and often perform poorly when they are directly evaluated on real sequences. Another promising direction is to learn from unlabeled videos, which are readily available

\footnotetext{
*Work mainly done during an internship at Tencent AI Lab. Copyright (C) 2019, Association for the Advancement of Artificial Intelligence (www.aaai.org). All rights reserved.
}

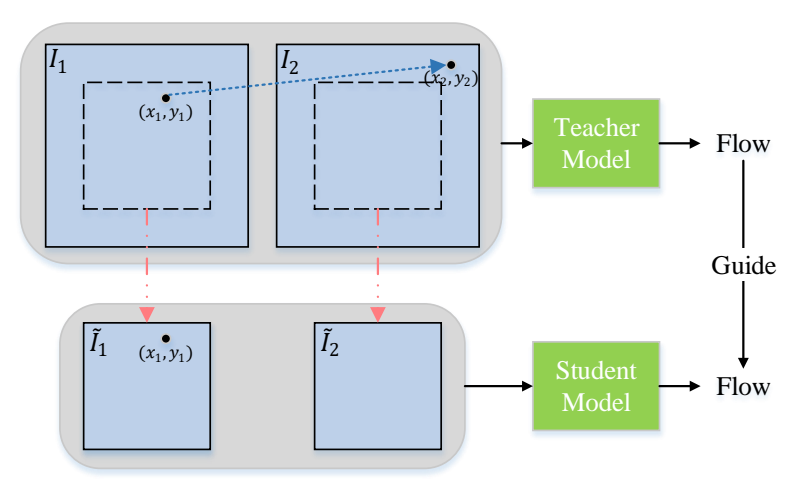

Figure 1: Data distillation illustration. We use the optical flow predictions from our teacher model to guide the learning of our student model.

at much larger scale. (Jason, Harley, and Derpanis 2016; Ren et al. 2017) employ the classical warping idea, and train $\mathrm{CNNs}$ with a photometric loss defined on the difference between reference and warped target images. Recent methods propose additional loss terms to cope with occluded pixels (Meister, Hur, and Roth 2018; Wang et al. 2018), or utilize multi-frames to reason occlusion (Janai et al. 2018). However, all these methods rely on hand-crafted energy terms to regularize optical flow estimation, lacking key capability to learn optical flow of occluded pixels. As a result, there is still a large performance gap comparing these methods with state-of-the-art fully supervised methods.

Is it possible to learn optical flow in a data-driven way, while not using any ground truth at all? In this paper, we address this issue by a data distilling approach. Our algorithm optimizes two models, a teacher model and a student model (as shown in Figure 1). We train the teacher model to estimate optical flow for non-occluded pixels (e.g., $\left(x_{1}, y_{1}\right)$ in $\left.I_{1}\right)$. Then, we hallucinate flow occlusion by cropping patches from original images (pixel $\left(x_{1}, y_{1}\right)$ now becomes occluded in $\widetilde{I}_{1}$ ). Predictions from our teacher model are used as annotations to directly guide the student network to learn optical flow. Both networks share the identical architecture, and are trained end-to-end with simple loss functions. The student network is used to produce optical flow at test time, and runs at real time. 


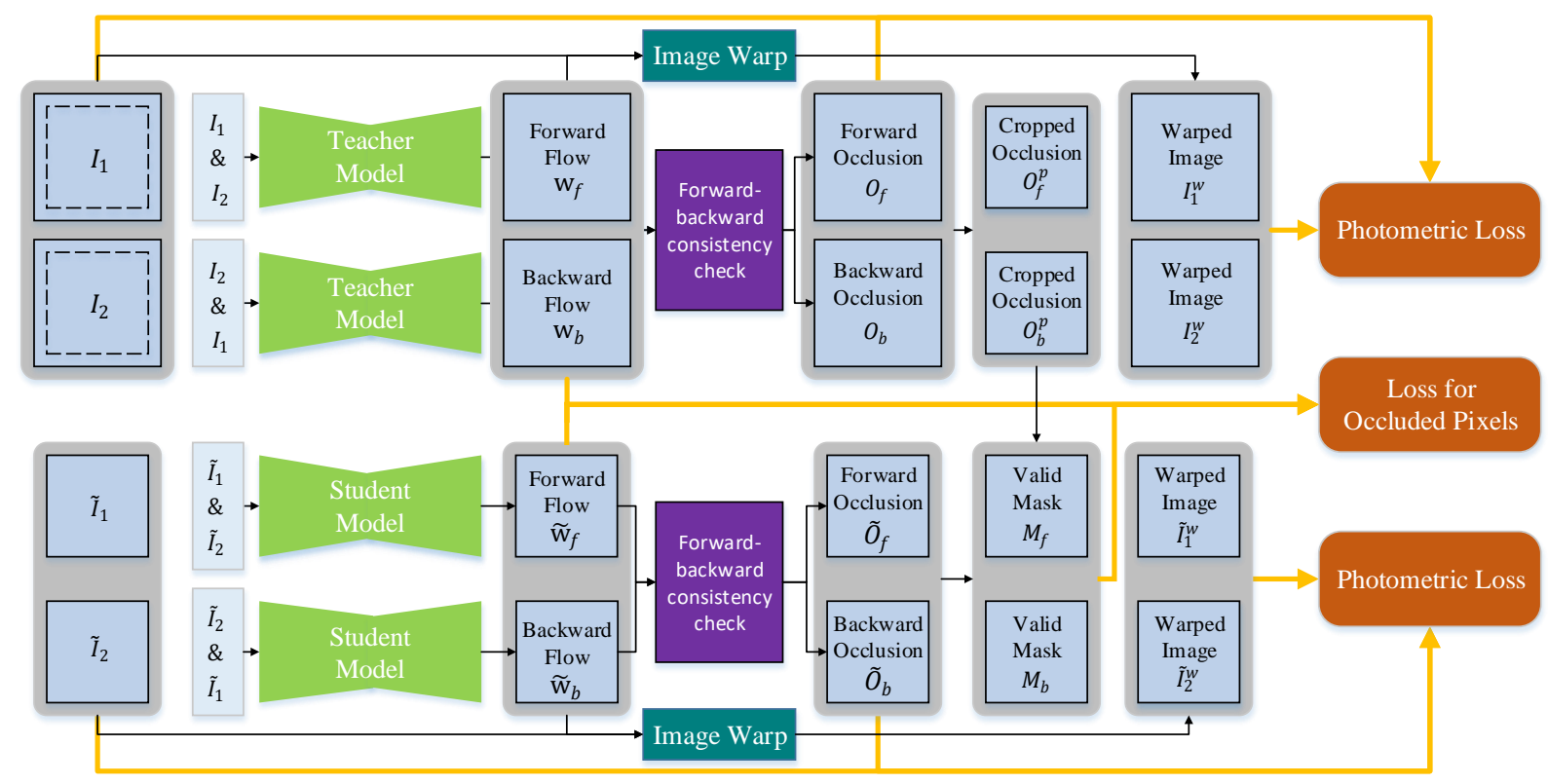

Figure 2: Framework overview of DDFlow. Our teacher model and student model have identical network structures. We train the teacher model with a photometric loss $L_{p}$ for non-occluded pixels. The student model is trained with both $L_{p}$ and $L_{o}$, a loss for occluded pixels. $L_{o}$ only functions on pixels that are non-occluded in original images but occluded in cropped patches (guided by Valid Mask $M_{f}, M_{b}$ ). During testing, only the student model is used.

The resulted self-training approach yields the highest accuracy among all unsupervised learning methods. At the time of writing, our method outperforms all published unsupervised flow methods on the Flying Chairs, MPI Sintel, KITTI 2012 and 2015 benchmarks. More notably, our method achieves a Fl-noc error of $4.57 \%$ on KITTI 2012, a Fl-all error of $14.29 \%$ on KITTI 2015, even outperforming several recent fully supervised methods which are fine-tuned for each dataset (Dosovitskiy et al. 2015; Ranjan and Black 2017; Bailer, Varanasi, and Stricker 2017; Zweig and Wolf 2017; Xu, Ranftl, and Koltun 2017).

\section{Related Work}

Optical flow estimation has been a long-standing challenge in computer vision. Early variational approaches (Horn and Schunck 1981; Sun, Roth, and Black 2010) formulate it as an energy minimization problem based on brightness constancy and spatial smoothness. Such methods are effective for small motion, but tend to fail when displacements are large.

Later, (Brox and Malik 2011; Weinzaepfel et al. 2013) integrate feature matching to tackle this issue. Specially, they find sparse feature correspondences to initialize flow estimation and further refine it in a pyramidal coarse-to-fine manner. The seminal work EpicFlow (Revaud et al. 2015) interpolates dense flow from sparse matches and has become a widely used post-processing pipeline. Recently, (Bailer, Varanasi, and Stricker 2017; Xu, Ranftl, and Koltun 2017) use convolutional neural networks to learn a feature embedding for better matching and have demonstrated superior performance. However, all of these classical methods are often time-consuming, and their modules usually involve special tuning for different datasets.

The success of deep neural networks has motivated the development of optical flow learning methods. The pioneer work is FlowNet (Dosovitskiy et al. 2015), which takes two consecutive images as input and outputs a dense optical flow map. The following FlowNet 2.0 (Ilg et al. 2017) significantly improves accuracy by stacking several basic FlowNet modules together, and iteratively refining them. SpyNet (Ranjan and Black 2017) proposes to warp images at multiple scales to handle large displacements, and introduces a compact spatial pyramid network to predict optical flow. Very recently, PWC-Net (Sun et al. 2018) and LiteFlowNet (Hui, Tang, and Loy 2018) propose to warp features extracted from CNNs rather than warp images over different scales. They achieve state-of-the-art results while keeping a much smaller model size. Though promising performance has been achieved, these methods require a large amount of labeled training data, which is particularly difficult to obtain for optical flow.

As a result, existing end-to-end deep learning based approaches (Dosovitskiy et al. 2015; Mayer et al. 2016; Janai et al. 2018) turn to utilize synthetic datasets for pretraining. Unfortunately, there usually exists a large domain gap between the distribution of synthetic datasets and natural scenes (Liu et al. 2008). Existing networks (Dosovitskiy et al. 2015; Ranjan and Black 2017) trained only on synthetic data turn to overfit, and often perform poorly when directly evaluated on real sequences. 


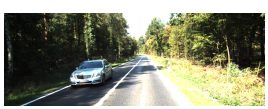

(a) $I_{1}$

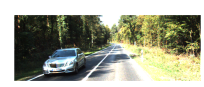

(g) $\widetilde{I}_{1}$

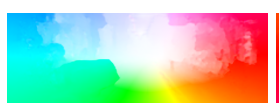

(b) $\mathrm{w}_{f}$

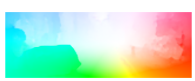

(h) $\widetilde{\mathrm{w}}_{f}$

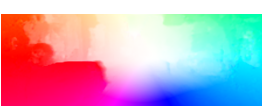

(c) $\mathrm{w}_{b}$

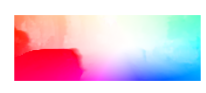

(i) $\widetilde{\mathrm{w}}_{b}$

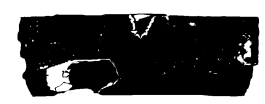

(d) $O_{f}$

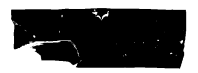

(j) $\widetilde{O}_{f}$

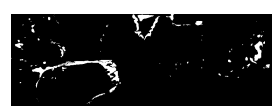

(e) $O_{b}$

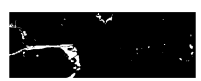

(k) $\widetilde{O}_{b}$

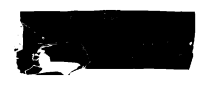

(f) $M_{f}$

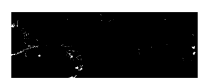

(1) $M_{b}$

Figure 3: Example intermediate results from DDFlow on KITTI. (a) is the first input image; (b,c) are forward and backward flow; (d,e) are forward and backward occlusion maps. $(\mathrm{g})$ is the cropped patch of (a); (h,i,j,k) are the corresponding forward flow, backward flow, forward occlusion map and backward occlusion map respectively. (f,l) are forward and backward valid mask, where 1 means the pixel is occluded in ( $\mathrm{g}$ ) but non-occluded in (a), 0 otherwise.

One promising direction is to develop unsupervised learning approaches. (Jason, Harley, and Derpanis 2016; Ren et al. 2017) construct loss functions based on brightness constancy and spatial smoothness. Specifically, the target image is warped according to the predicted flow, and then the difference between the reference image and the warped image is optimized using a photometric loss. Unfortunately, this loss would provide misleading information when the pixels are occluded.

Very recently, (Meister, Hur, and Roth 2018; Wang et al. 2018) propose to first reason occlusion map and then exclude those occluded pixels when computing the photometric difference. Most recently, (Janai et al. 2018) introduce an unsupervised framework to estimate optical flow using a multi-frame formulation with temporal consistency. This method utilizes more data with more advanced occlusion reasoning, and hence achieves more accurate results. However, all these unsupervised learning methods rely on handcrafted energy terms to guide optical flow estimation, lacking key capability to learn optical flow of occluded pixels. As a consequence, the performance is still a large gap compared with state-of-the-art supervised methods.

To bridge this gap, we propose to perform knowledge distillation from unlabeled data, inspired by (Hinton, Vinyals, and Dean 2015; Radosavovic et al. 2018) which performed knowledge distillation from multiple models or labeled data. In contrast to previous knowledge distillation methods, we do not use any human annotations. Our idea is to generate annotations on unlabeled data using a model trained with a classical optical flow energy, and then retrain the model using those extra generated annotations. This yields a simple yet effective method to learn optical flow for occluded pixels in a totally unsupervised manner.

\section{Method}

We first illustrate our learning framework in Figure 2. We simultaneously train two CNNs (a teacher model and a student model) with the same structure. The teacher model is employed to predict optical flow for non-occluded pixels and the student model is used to predict optical flow of both nonoccluded and occluded pixels. During testing time, only the student model is used to produce optical flow. Before describing our method, we define our notations as follows.

\section{Notation}

For our teacher model, we denote $I_{1}, I_{2} \in \mathbb{R}^{H \times W \times 3}$ for two consecutive RGB images, where $H$ and $W$ are height and width respectively. Our goal is to estimate a forward optical flow $\mathrm{w}_{f} \in \mathbb{R}^{H \times W \times 2}$ from $I_{1}$ to $I_{2}$. After obtaining $\mathrm{w}_{f}$, we can warp $I_{2}$ towards $I_{1}$ to get a warped image $I_{2}^{w}$. Here, we also estimate a backward optical flow $\mathrm{w}_{b}$ from $I_{2}$ to $I_{1}$ and a backward warp image $I_{1}^{w}$. Since there are many cases where one pixel is only visible in one image but not visible in the other image, namely occlusion, we denote $O_{f}$, $O_{b} \in \mathbb{R}^{H \times W \times 1}$ as the forward and backward occlusion map respectively. For $O_{f}$ and $O_{b}$, value 1 means that the pixel in that location is occluded, while value 0 means not occluded.

Our student model follows similar notations. We distill consistent predictions ( $\mathrm{w}_{f}$ and $\left.O_{f}\right)$ from our teacher model, and crop patches on the original images to hallucinate occlusion. Let $\widetilde{I}_{1}, \widetilde{I}_{2}, \mathrm{w}_{f}^{p}, \mathrm{w}_{b}^{p}, O_{f}^{p}$ and $O_{b}^{p}$ denote the cropped image patches of $I_{1}, I_{2}, \mathrm{w}_{f}, \mathrm{w}_{b}, O_{f}$ and $O_{b}$ respectively. The cropping size is $h \times w$, where $h<H, w<W$.

The student network takes $\widetilde{I}_{1}, \widetilde{I}_{2}$ as input, and produces a forward and backward flow, a warped image, a occlusion $\operatorname{map} \widetilde{\mathrm{w}}_{f}, \widetilde{\mathrm{w}}_{b}, \widetilde{I}_{2}^{w}, \widetilde{I}_{1}^{w}, \widetilde{O}_{f}, \widetilde{O}_{b}$ respectively.

After obtaining $O_{f}^{p}$ and $\widetilde{O}_{f}$, we compute another mask $M_{f}$, where value 1 means the pixel is occluded in image patch $\widetilde{I}_{1}$ but non-occluded in the original image $I_{1}$. The backward mask $M_{b}$ is computed in the same way. Figure 3 shows a real example for each notation used in DDFlow.

\section{Network Architecture}

In principle, DDFlow can use any backbone network to learn optical flow. We select PWC-Net (Sun et al. 2018) as our backbone network due to its remarkable performance and compact model size. PWC-Net learns 7-level feature representations for two input images, and gradually conducts feature warping and cost volume construction from the last level to the third level. As a result, the output resolution of flow map is a quarter of the original image size. We upsample the output flow to the full resolution using bilinear interpolation. To train two networks simultaneously in a totally unsupervised way, we normalize features when constructing cost volume, and swap the image pairs in our input to produce both forward and backward flow. 
(a) Input Image 1

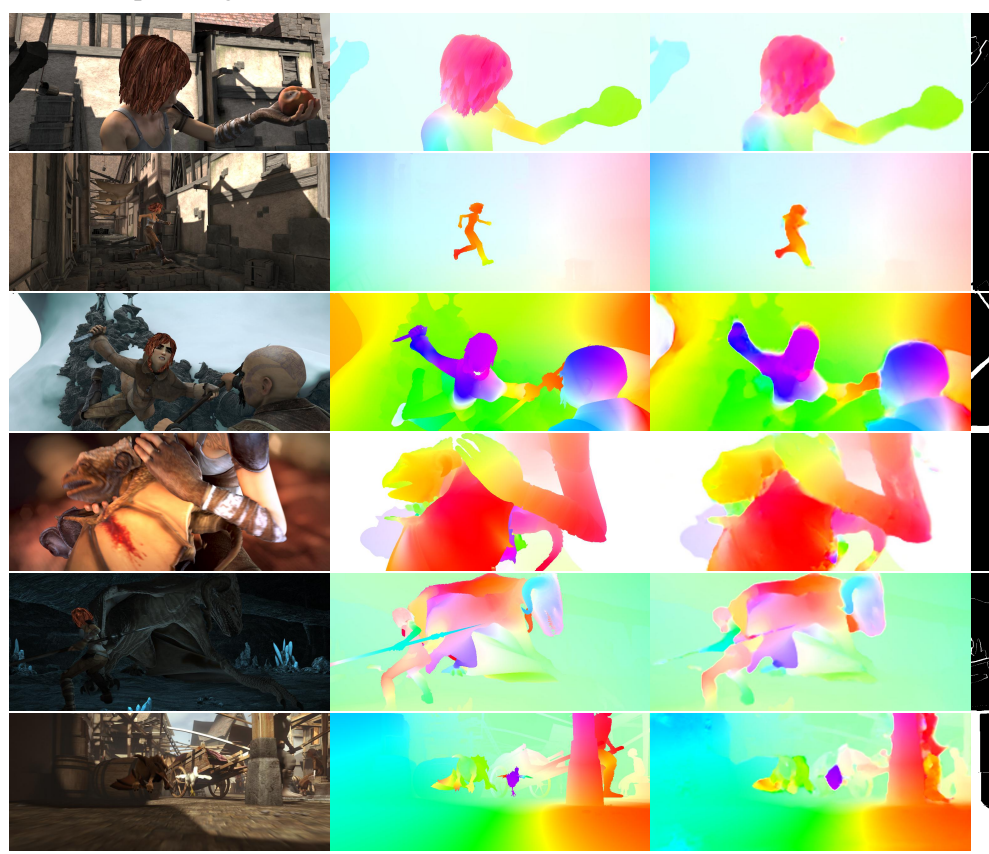

(d) GT Occlusion

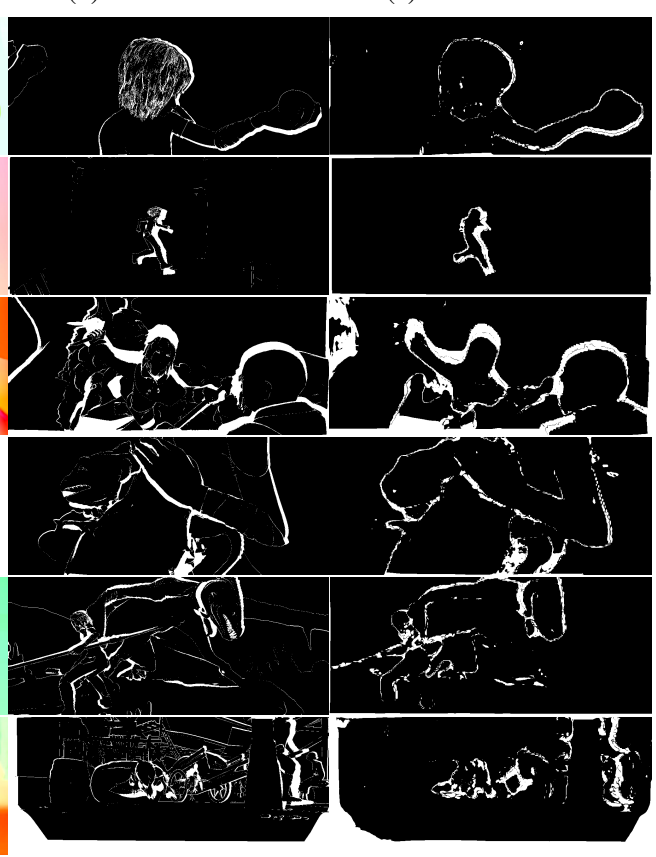

Figure 4: Sample results on Sintel datasets. The first three rows are from Sintel Clean, while the last three are from Sintel Final. Our method estimates accurate optical flow and reliable occlusion maps.

We use the identical network architecture for our teacher and student model. The only difference between them is to train each with different input data and loss functions. Next, we discuss how to generate such data, and construct loss functions for each model in detail.

\section{Unlabeled Data Distillation}

For prior unsupervised optical flow learning methods, the only guidance is a photometric loss which measures the difference between the reference image and the warped target image. However, photometric loss makes no sense for occluded pixels. To tackle this issue, We distill predictions from our teacher model, and use them to generate input/output data for our student model. Figure 1 shows a toy example for our data distillation idea.

Suppose pixel $\left(x_{2}, y_{2}\right)$ in $I_{2}$ is the corresponding pixel of $\left(x_{1}, y_{1}\right)$ in $I_{1}$. Given $\left(x_{1}, y_{1}\right)$ is non-occluded, we can use the classical photometric loss to find its optical flow using our teacher model. Now, if we crop image patches $\widetilde{I}_{1}$ and $\widetilde{I}_{2}$, pixel $\left(x_{1}, y_{1}\right)$ in $\widetilde{I}_{1}$ becomes occluded, since there is no corresponding pixel in $\widetilde{I}_{2}$ any more. Fortunately, the optical flow prediction for $\left(x_{1}, y_{1}\right)$ from our teacher model is still there. We then directly use this prediction as annotation to guide the student model to learn optical flow for the occluded pixel $\left(x_{1}, y_{1}\right)$ in $\widetilde{I}_{1}$. This is the key intuition behind DDFlow.

Figure 2 shows the main data flow for our approach. To make full use of the input data, we compute both forward and backward flow $\mathrm{w}_{f}, \mathrm{w}_{b}$ for the original frames, as well as their warped images $I_{1}^{w}, I_{2}^{w}$. We also estimate two occlusion maps $O_{f}, O_{w}$ by checking forward-backward consistency. The teacher model is trained with a photometric loss, which minimizes a warping error using $I_{1}, I_{2}, O_{f}, O_{w}, I_{1}^{w}, I_{2}^{w}$. This model produces accurate optical flow predictions for non-occluded pixels in $I_{1}$ and $I_{2}$.

For our student model, we randomly crop image patches $\widetilde{I}_{1}, \widetilde{I}_{2}$ from $I_{1}, I_{2}$, and we compute forward and backward flow $\widetilde{\mathrm{w}}_{f}, \widetilde{\mathrm{w}}_{b}$ for them. A similar photometric loss is employed for the non-occluded pixels in $\widetilde{I}_{1}$ and $\widetilde{I}_{2}$. In addition, predictions from our teacher model are employed as output annotations to guide those pixels occluded in cropped image patches but non-occluded in original images. Next, we discuss how to construct all the loss functions.

\section{Loss Functions}

Our loss functions include two components: photometric loss $L_{p}$ and loss for occluded pixels $L_{o}$. Optionally, smoothness losses can also be added. Here, we focus on the above two loss terms for simplicity. For the teacher model, only $L_{p}$ is used to estimate the flow of non-occluded pixels, while for student model, $L_{p}$ and $L_{o}$ are both employed to estimate the optical flow of non-occluded and occluded pixels.

Occlusion Estimation. Our occlusion detection is based on the forward-backward consistency prior (Sundaram, Brox, and Keutzer 2010; Meister, Hur, and Roth 2018). That is, for non-occluded pixels, the forward flow should be the inverse of the backward flow at the corresponding pixel in the second image. We consider pixels as occluded when the mismatch between forward flow and backward flow is too large or the flow is out of image boundary $\Omega$. Take a forward 


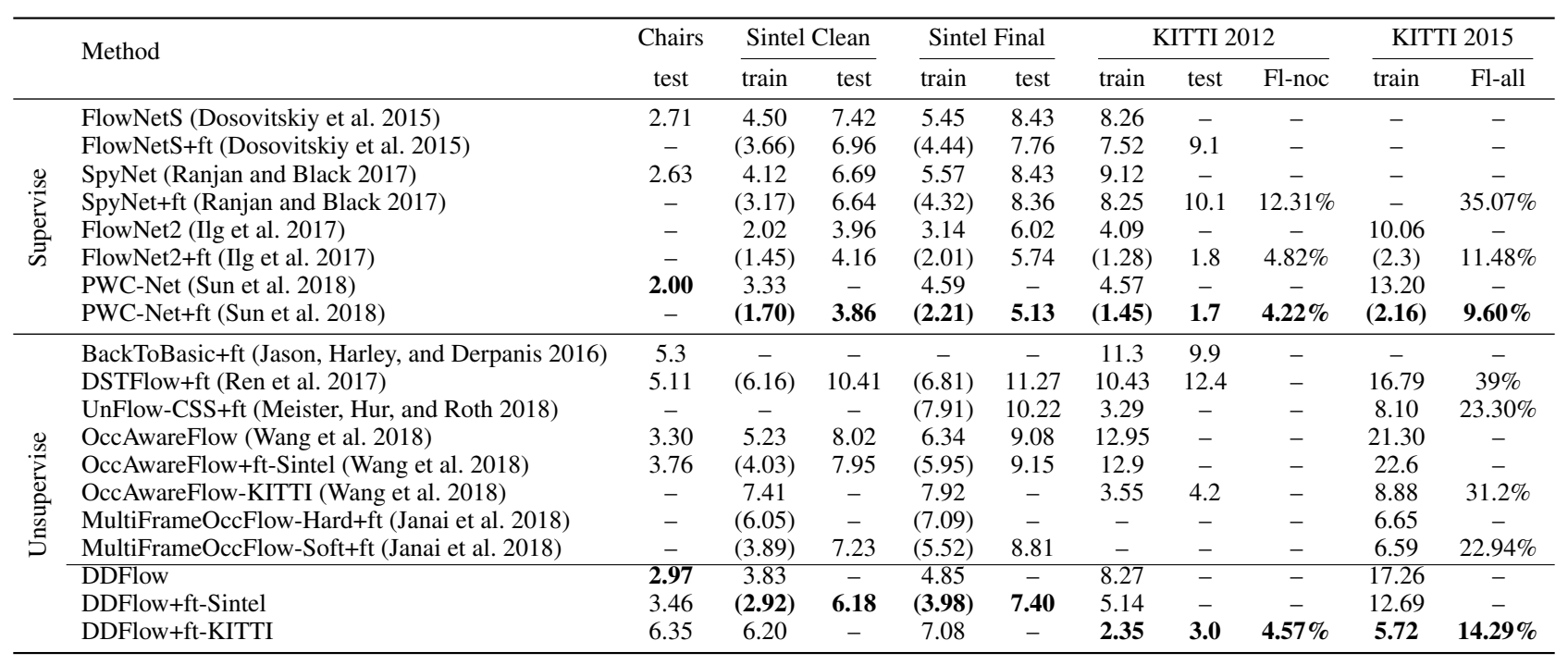

Table 1: Comparison to state-of-the-art optical flow estimation methods. All numbers are EPE except for the last column of KITTI 2012 and KITTI 2015 test sets, where we report percentage of erroneous pixels (Fl). Missing entries (-) indicate that the results are not reported for the respective method. Parentheses mean that the training is performed on the same dataset. Bold fonts highlight the best results among supervised and unsupervised methods respectively. Note that MultiFrameOccFlow (Janai et al. 2018) utilizes multiple frames, while all other methods use only two consecutive frames.

occlusion map as an example, we first compute the reversed forward flow $\hat{\mathrm{w}}_{f}=\mathrm{w}_{b}\left(\mathbf{p}+\mathrm{w}_{f}(\mathbf{p})\right)$, where $\mathbf{p} \in \Omega$. A pixel is considered occluded if either of the following constraints is violated:

$$
\left\{\begin{array}{l}
\left|\mathrm{w}_{f}+\hat{\mathrm{w}}_{f}\right|^{2}<\alpha_{1}\left(\left|\mathrm{w}_{f}\right|^{2}+\left|\hat{\mathrm{w}}_{f}\right|^{2}\right)+\alpha_{2}, \\
\mathbf{p}+\mathrm{w}_{f}(\mathbf{p}) \notin \Omega,
\end{array}\right.
$$

where we set $\alpha_{1}=0.01, \alpha_{2}=0.05$ for all our experiments. Backward occlusion maps are computed in the same way.

Photometric Loss. The photometric loss is based on the brightness constancy assumption, which measures the difference between the reference image and the warped target image. It is only effective for non-occluded pixels. We define a simple loss as follows:

$$
\begin{aligned}
L_{p}= & \sum \psi\left(I_{1}-I_{2}^{w}\right) \odot\left(1-O_{f}\right) / \sum\left(1-O_{f}\right) \\
& +\sum \psi\left(I_{2}-I_{1}^{w}\right) \odot\left(1-O_{b}\right) / \sum\left(1-O_{b}\right)
\end{aligned}
$$

where $\psi(x)=(|x|+\epsilon)^{q}$ is a robust loss function, $\odot$ denotes the element-wise multiplication. During our experiments, we set $\epsilon=0.01, q=0.4$. Our teacher model only minimizes this loss.

Loss for Occluded Pixels. The key element in unsupervised learning is the loss for occluded pixels. In contrast to existing loss functions relying on smoothing prior to constrain flow estimation, our loss is purely data-driven. This enables us to directly learn from real data, and produce more accurate flow. To this end, we define our loss on pixels that are occluded in the cropped patch but non-occluded in the original image. Then, supervision is generated using predictions of the original image from our teacher model, which produces reliable optical flow for non-occluded pixels.
To find these pixels, we first compute a valid mask $M$ representing the pixels that are occluded in the cropped image but non-occluded in the original image:

$$
M_{f}=\operatorname{clip}\left(\widetilde{O}_{f}-O_{f}^{p}, 0,1\right)
$$

Backward mask $M_{b}$ is computed in the same way. Then we define our loss for occluded pixels in the following,

$$
\begin{aligned}
L_{o}=\sum \psi\left(\mathrm{w}_{f}^{p}\right. & \left.-\widetilde{\mathrm{w}}_{f}\right) \odot M_{f} / \sum M_{f} \\
& +\sum \psi\left(\mathrm{w}_{b}^{p}-\widetilde{\mathrm{w}}_{b}\right) \odot M_{b} / \sum M_{b}
\end{aligned}
$$

We use the same robust loss function $\psi(x)$ with the same parameters defined in Eq. 2. Our student model minimizes the simple combination $L_{p}+L_{o}$.

\section{Experiments}

We evaluate DDFlow on standard optical flow benchmarks including Flying Chairs (Dosovitskiy et al. 2015), MPI Sintel (Butler et al. 2012), KITTI 2012(Geiger, Lenz, and Urtasun 2012), and KITTI 2015 (Menze and Geiger 2015). We compare our results with state-of-the-art unsupervised methods including BackToBasic(Jason, Harley, and Derpanis 2016), DSTFlow(Ren et al. 2017), UnFlow(Meister, Hur, and Roth 2018), OccAwareFlow(Wang et al. 2018) and MultiFrameOccFlow(Janai et al. 2018), as well as fully supervised learning methods including FlowNet(Dosovitskiy et al. 2015), SpyNet(Ranjan and Black 2017), FlowNet2(Ilg et al. 2017) and PWC-Net(Sun et al. 2018). Note that MultiFrameOccFlow (Janai et al. 2018) utilizes multiple frames as input, while all other methods use only two consecutive frames. To ensure reproducibility and advance further innovations, we make our code and models publicly available our our project website. 
(a) Input Image 1

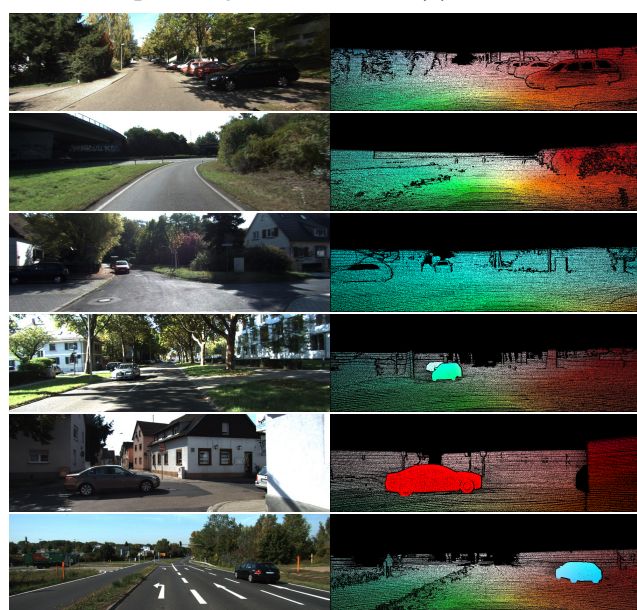

(c) Our Flow

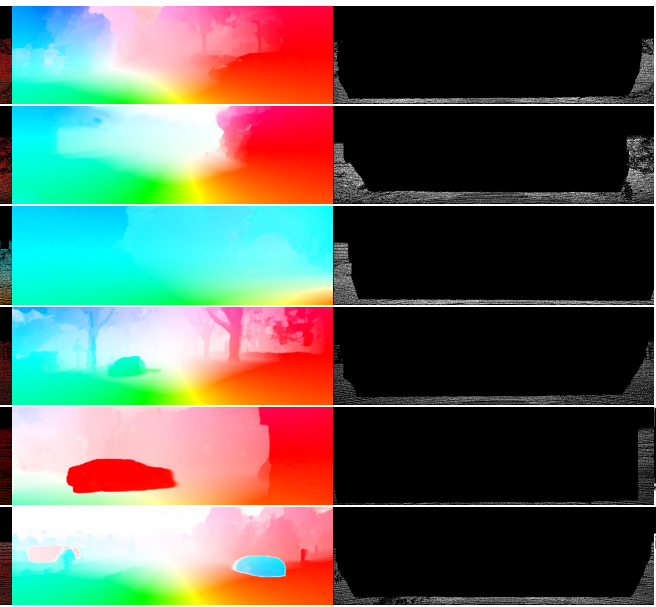

(e) Our Occlusion

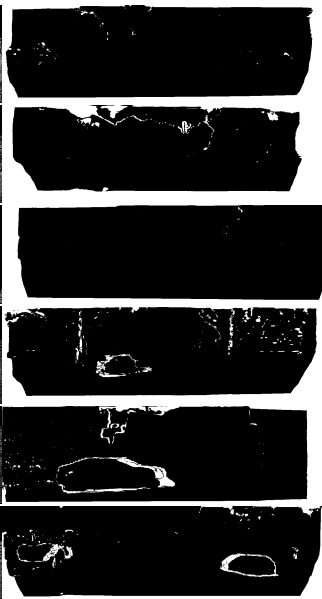

Figure 5: Example results on KITTI datasets. The first three rows are from KITTI 2012, and the last three are from KITTI 2015. Our method estimates accurate optical flow and reliable occlusion maps. Note that on KITTI datasets, the occlusion masks are sparse and only contain pixels moving out of the image boundary.

\section{Implementation Details}

Data Preprocessing. We preprocess the image pairs using census transform (Zabih and Woodfill 1994), which is proved to be robust for optical flow estimation (Hafner, Demetz, and Weickert 2013). We find that this simple procedure can indeed improve the performance of unsupervised optical flow estimation, which is consistent with (Meister, Hur, and Roth 2018).

Training procedure. For all our experiments, we use the same network architecture and train our model using Adam optimizer (Kingma and Ba 2014) with $\beta_{1}=0.9$ and $\beta_{2}=0.999$. For all datasets, we set batch size as 4 . For all individual experiments, we use a initial learning rate of 1e-4, and it decays half every 50k iterations. For data augmentation, we only use random cropping, random flipping, and random channel swapping. Thanks to the simplicity of our loss functions, there is no need to tune hyper-parameters.

Following prior work, we first pre-train DDFlow on Flying Chairs. We initialize our teacher network from random, and warm it up with 200k iterations using our photometric loss without considering occlusion. Then, we add our occlusion detection check, and train the network with the photometric loss $L_{p}$ for another 300k iterations. After that, we initialize the student model with the weights from our teacher model, and train both the teacher model (with $L_{p}$ ) and the student model (with $L_{p}+L_{o}$ ) together for $300 \mathrm{k}$ iterations. This concludes our pre-training, and the student model is used for future fine-tuning.

We use the same fine-tuning procedure for all Sintel and KITTI datasets. First, we initialize the teacher network using the pre-trained student model from Flying Chairs, and train it for 300k iterations. Then, similar to pre-training on Flying Chairs, the student network is initialized with the new teacher model, and both networks are trained together for another $300 \mathrm{k}$ iterations. The student model is used during our evaluation.
Evaluation Metrics. We consider two widely-used metrics to evaluate optical flow estimation and one metric of occlusion evaluation: average endpoint error (EPE), percentage of erroneous pixels ( $\mathrm{Fl})$, harmonic average of the precision and recall (F-measure). We also report the results of EPE over non-occluded pixels (NOC) and occluded pixels (OCC) respectively. EPE is the ranking metric on MPI Sintel benchmark, and Fl is the ranking metric on KITTI benchmarks.

\section{Comparison to State-of-the-art}

We compare our results with state-of-the art methods in Table 1. As we can see, our approach, DDFlow, outperforms all existing unsupervised flow learning methods on all datasets. On the test set of Flying Chairs, our EPE is better than all prior results, decreasing from previous state-of-the-art 3.30 to 2.97. More importantly, simply evaluating our model only pre-trained on Flying Chairs, DDFlow achieves EPE $=3.83$ on Sintel Clean and EPE=4.85 on Sintel Final, which are even better than the results from state-of-the-art unsupervised methods (Wang et al. 2018; Janai et al. 2018) finetuned specifically for Sintel. This is remarkable, as it shows the great generalization capability of DDFlow.

After we finetuned DDFlow using frames from the Sintel training set, we achieved an EPE $=7.40$ on the Sintel Final testing benchmark, improving the best prior result $(\mathrm{EPE}=8.81$ from (Janai et al. 2018) ) by a relative margin of $14.0 \%$. Similar improvement (from 7.23 to 6.18 ) is also observed on the Sintel Clean testing benchmark. Our model is even better than some supervised methods including (Dosovitskiy et al. 2015) and (Ranjan and Black 2017), which are finetuned on Sintel using ground truth annotations. Figure 4 shows sample DDFlow results from Sintel, comparing our optical flow estimations and occlusion masks with the ground truth.

On KITTI dataset, the improvement from DDFlow is even 


\begin{tabular}{|c|c|c|c|c|c|c|c|c|c|c|c|c|c|c|c|}
\hline \multirow{2}{*}{$\begin{array}{l}\text { Occlusion } \\
\text { Handling }\end{array}$} & \multirow{2}{*}{$\begin{array}{c}\text { Census } \\
\text { Transform }\end{array}$} & \multirow{2}{*}{$\begin{array}{c}\text { Data } \\
\text { Distillation }\end{array}$} & \multirow{2}{*}{$\begin{array}{l}\text { Chairs } \\
\text { ALL }\end{array}$} & \multicolumn{3}{|c|}{ Sintel Clean } & \multicolumn{3}{|c|}{ Sintel Final } & \multicolumn{3}{|c|}{ KITTI 2012} & \multicolumn{3}{|c|}{ KITTI 2015} \\
\hline & & & & ALL & NOC & $\overline{\mathrm{OCC}}$ & ALL & $\mathrm{NOC}$ & $\overline{\mathrm{OCC}}$ & $\overline{\mathrm{ALL}}$ & NOC & $\overline{\mathrm{OCC}}$ & $\overline{\mathrm{ALL}}$ & NOC & $\overline{\mathrm{OCC}}$ \\
\hline$x$ & $x$ & $x$ & 4.06 & $(5.05)$ & $(2.45)$ & (38.09) & (7.54) & $(4.81)$ & $(42.46)$ & 10.76 & 3.35 & 59.86 & 16.85 & 6.45 & 82.64 \\
\hline$\checkmark$ & $x$ & $x$ & 3.95 & (4.45) & (2.16) & (33.48) & (6.56) & (4.12) & (37.83) & 6.67 & 1.94 & 38.01 & 12.42 & 5.67 & 60.59 \\
\hline$x$ & $\checkmark$ & $x$ & 3.75 & (3.90) & (1.60) & (33.31) & $(5.23)$ & $(2.80)$ & $(36.35)$ & 8.66 & 1.47 & 56.24 & 14.04 & 4.06 & 77.16 \\
\hline$\checkmark$ & $\checkmark$ & $x$ & 3.24 & (3.37) & (1.34) & (29.36) & (4.47) & (2.32) & (31.86) & 4.50 & 1.10 & 27.04 & 8.01 & 3.02 & 42.66 \\
\hline$\checkmark$ & $\checkmark$ & $\checkmark$ & 2.97 & (2.92) & (1.27) & (23.92) & (3.98) & (2.21) & (26.74) & 2.35 & 1.02 & 11.31 & 5.72 & 2.73 & 24.68 \\
\hline
\end{tabular}

Table 2: Ablation study. We compare the results of EPE over all pixels (ALL), non-occluded pixels (NOC) and occluded pixels (OCC) under different settings. Bold fonts highlight the best results.

\begin{tabular}{lcccc}
\hline \multirow{2}{*}{ Method } & $\begin{array}{c}\text { Sintel } \\
\text { Clean }\end{array}$ & $\begin{array}{c}\text { Sintel } \\
\text { Final }\end{array}$ & $\begin{array}{c}\text { KITTI } \\
2012\end{array}$ & $\begin{array}{c}\text { KITTI } \\
2015\end{array}$ \\
\hline MODOF & - & 0.48 & - & - \\
OccAwareFlow-ft & $(0.54)$ & $(0.48)$ & $\mathbf{0 . 9 5}^{*}$ & $0.88^{*}$ \\
MultiFrameOccFlow-Soft+ft & $(0.49)$ & $(0.44)$ & - & $\mathbf{0 . 9 1}^{*}$ \\
Ours & $\mathbf{( 0 . 5 9 )}$ & $\mathbf{( 0 . 5 2 )}$ & $0.94^{*}$ & $0.86^{*}$ \\
\hline
\end{tabular}

Table 3: Comparison to state-of-the-art occlusion estimation methods. ${ }^{*}$ marks cases where the occlusion map is sparse and only the annotated pixels are considered.

more significant. On KITTI 2012 testing set, DDFlow yields an $\mathrm{EPE}=3.0,28.6 \%$ lower than the best existing counterpart (EPE=4.2 from (Wang et al. 2018)). For the ranking measurement on KITTI 2012, we achieve Fl-noc $=4.57 \%$, even better than the result (4.82 \%) from the well-known FlowNet 2.0. For KITTI 2015, DDFlow performs particularly well. The Fl-all from DDFlow reaches $14.29 \%$, not only better than the best unsupervised method by a large margin (37.7\% relative improvement), but also outperforming several recent fully supervised learning methods including (Ranjan and Black 2017; Bailer, Varanasi, and Stricker 2017; Zweig and Wolf 2017; Xu, Ranftl, and Koltun 2017). Example results from KITTI 2012 and 2015 can be seen in Figure 5.

\section{Occlusion Estimation}

Next, we evaluate our occlusion estimation on both Sintel and KITTI dataset. We compare our method with MODOF(Xu, Jia, and Matsushita 2012), OccAwareFlow$\mathrm{ft}($ Wang et al. 2018), MultiFrameOccFlow-Soft+ft(Janai et al. 2018) using F-measure. Note KITTI datasets only have sparse occlusion map.

As shown in Table 3, our method achieve best occlusion estimation performance on Sintel Clean and Sintel Final datasets over all competing methods. On KITTI dataset, the ground truth occlusion masks only contain pixels moving out of the image boundary. However, our method will also estimate the occlusions within the image range. Under such settings, our method can achieve comparable performance.

\section{Ablation Study}

We conduct a thorough ablation analysis for different components of DDflow. We report our findings in Table 2.

Occlusion Handling. Comparing the first row and the second row, the third row and the fourth row, we can see that occlusion handling can improve the optical flow estima- tion performance over all pixels, non-occluded pixels and occluded pixels on all datasets. It is because that brightness constancy assumption does not hold for occluded pixels.

Census Transform. Census transform can compensate for illumination changes, which is robust for optical flow estimation and has been widely used in traditional methods. Comparing the first row and the third row, the second row and the fourth row, we can see that it indeed constantly improves the performance on all datasets.

Data Distillation. Since brightness constancy assumption does not hold for occluded pixels and there is no ground truth flow for occluded pixels, we introduce a data distillation loss to address this problem. As shown in the fourth row and the fifth row, occluded prediction can improve the performance on all datasets, especially for occluded pixels. EPE-OCC decreases from 29.36 to 23.93 (by $18.5 \%$ ) on Sintel Clean, from 31.86 to 26.74 (by $16.1 \%$ ) on Sintel Final dataset, from 27.04 to 11.31 (by $58.2 \%$ ) on KITTI 2012 and from 42.66 to 24.68 (by $42.1 \%$ ) on KITTI 2015. Such a big improvement demonstrates the effectiveness of DDFlow.

Our distillation strategy works particularly well near image boundary, since our teacher model can distill reliable labels for these pixels. For occluded pixels elsewhere, our method is not as effective, but still produces reasonable results to some extent. This is because we crop at random location for student model, which covers a large amount of occlusions. Exploring new ideas to cope with occluded pixels at any location can be a promising research direction in the future.

\section{Conclusion}

We have presented a data distillation approach to learn optical flow from unlabeled data. We have shown that CNNs can be self-trained to estimate optical flow, even for occluded pixels, without using any human annotations. To this end, we construct two networks. The predictions from the teacher network are used as annotations to guide the student network to learn optical flow. Our method, DDFlow, has achieved the highest accuracy among all prior unsupervised methods on all challenging optical flow benchmarks. Our work makes a step towards distilling optical flow knowledge from unlabeled data. Going forward, our results suggest that our data distillation technique may be a promising direction for advancing other vision tasks like stereo matching (Zbontar and LeCun 2016) or depth estimation (Eigen, Puhrsch, and Fergus 2014). 


\section{References}

Bailer, C.; Varanasi, K.; and Stricker, D. 2017. Cnn-based patch matching for optical flow with thresholded hinge embedding loss. In CVPR.

Bonneel, N.; Tompkin, J.; Sunkavalli, K.; Sun, D.; Paris, S.; and Pfister, H. 2015. Blind video temporal consistency. ACM Trans. Graph. 34(6):196:1-196:9.

Brox, T., and Malik, J. 2011. Large displacement optical flow: descriptor matching in variational motion estimation. TPAMI 33(3):500-513.

Brox, T.; Bruhn, A.; Papenberg, N.; and Weickert, J. 2004. High accuracy optical flow estimation based on a theory for warping. In $E C C V$.

Butler, D. J.; Wulff, J.; Stanley, G. B.; and Black, M. J. 2012. A naturalistic open source movie for optical flow evaluation. In $E C C V$.

Chauhan, A. K., and Krishan, P. 2013. Moving object tracking using gaussian mixture model and optical flow. International Journal of Advanced Research in Computer Science and Software Engineering 3(4).

Dosovitskiy, A.; Fischer, P.; Ilg, E.; Hausser, P.; Hazirbas, C.; Golkov, V.; Van Der Smagt, P.; Cremers, D.; and Brox, T. 2015. Flownet: Learning optical flow with convolutional networks. In ICCV.

Eigen, D.; Puhrsch, C.; and Fergus, R. 2014. Depth map prediction from a single image using a multi-scale deep network. In NIPS.

Geiger, A.; Lenz, P.; and Urtasun, R. 2012. Are we ready for autonomous driving? the kitti vision benchmark suite. In CVPR.

Hafner, D.; Demetz, O.; and Weickert, J. 2013. Why is the census transform good for robust optic flow computation? In International Conference on Scale Space and Variational Methods in Computer Vision.

Hinton, G.; Vinyals, O.; and Dean, J. 2015. Distilling the knowledge in a neural network. arXiv preprint arXiv: 1503.02531 .

Horn, B. K., and Schunck, B. G. 1981. Determining optical flow. Artificial intelligence 17(1-3):185-203.

Hui, T.-W.; Tang, X.; and Loy, C. C. 2018. Liteflownet: A lightweight convolutional neural network for optical flow estimation. In $C V P R$.

Ilg, E.; Mayer, N.; Saikia, T.; Keuper, M.; Dosovitskiy, A.; and Brox, T. 2017. Flownet 2.0: Evolution of optical flow estimation with deep networks. In CVPR.

Janai, J.; Güney, F.; Ranjan, A.; Black, M. J.; and Geiger, A. 2018. Unsupervised learning of multi-frame optical flow with occlusions. In $E C C V$.

Jason, J. Y.; Harley, A. W.; and Derpanis, K. G. 2016. Back to basics: Unsupervised learning of optical flow via brightness constancy and motion smoothness. In ECCV.

Kingma, D. P., and Ba, J. 2014. Adam: A method for stochastic optimization. arXiv preprint arXiv:1412.6980.

Liu, C.; Freeman, W. T.; Adelson, E. H.; and Weiss, Y. 2008. Human-assisted motion annotation. In CVPR.
Mayer, N.; Ilg, E.; Hausser, P.; Fischer, P.; Cremers, D.; Dosovitskiy, A.; and Brox, T. 2016. A large dataset to train convolutional networks for disparity, optical flow, and scene flow estimation. In $C V P R$.

Meister, S.; Hur, J.; and Roth, S. 2018. UnFlow: Unsupervised learning of optical flow with a bidirectional census loss. In $A A A I$.

Menze, M., and Geiger, A. 2015. Object scene flow for autonomous vehicles. In CVPR.

Radosavovic, I.; Dollár, P.; Girshick, R.; Gkioxari, G.; and He, K. 2018. Data distillation: Towards omni-supervised learning. CVPR.

Ranjan, A., and Black, M. J. 2017. Optical flow estimation using a spatial pyramid network. In CVPR.

Ren, Z.; Yan, J.; Ni, B.; Liu, B.; Yang, X.; and Zha, H. 2017. Unsupervised deep learning for optical flow estimation. In AAAI.

Revaud, J.; Weinzaepfel, P.; Harchaoui, Z.; and Schmid, C. 2015. Epicflow: Edge-preserving interpolation of correspondences for optical flow. In CVPR.

Simonyan, K., and Zisserman, A. 2014. Two-stream convolutional networks for action recognition in videos. In NIPS.

Sun, D.; Yang, X.; Liu, M.-Y.; and Kautz, J. 2018. Pwcnet: Cnns for optical flow using pyramid, warping, and cost volume. In CVPR.

Sun, D.; Roth, S.; and Black, M. J. 2010. Secrets of optical flow estimation and their principles. In CVPR.

Sundaram, N.; Brox, T.; and Keutzer, K. 2010. Dense point trajectories by gpu-accelerated large displacement optical flow. In $E C C V$.

Wang, Y.; Yang, Y.; Yang, Z.; Zhao, L.; and Xu, W. 2018. Occlusion aware unsupervised learning of optical flow. In CVPR.

Weinzaepfel, P.; Revaud, J.; Harchaoui, Z.; and Schmid, C. 2013. Deepflow: Large displacement optical flow with deep matching. In $I C C V$.

Xu, L.; Jia, J.; and Matsushita, Y. 2012. Motion detail preserving optical flow estimation. TPAMI 34(9):1744-1757.

Xu, J.; Ranftl, R.; and Koltun, V. 2017. Accurate Optical Flow via Direct Cost Volume Processing. In CVPR.

Zabih, R., and Woodfill, J. 1994. Non-parametric local transforms for computing visual correspondence. In ECCV. Zbontar, J., and LeCun, Y. 2016. Stereo matching by training a convolutional neural network to compare image patches. JMLR 17(1-32):2.

Zweig, S., and Wolf, L. 2017. Interponet, a brain inspired neural network for optical flow dense interpolation. In $C V P R$. 